\title{
A powerful notch filter for PLI cancelation
}

This paper was downloaded from TechRxiv (https://www.techrxiv.org).

\section{LICENSE}

CC BY-NC-SA 4.0

SUBMISSION DATE / POSTED DATE

$28-11-2021$ / 09-12-2021

CITATION

Mobaien, Ali; Kheirati Roonizi, Arman; Boostani, Reza (2021): A powerful notch filter for PLI cancelation. TechRxiv. Preprint. https://doi.org/10.36227/techrxiv.17088890.v1

$\mathrm{DOI}$

10.36227/techrxiv.17088890.v1 


\section{A powerful notch filter for PLI cancelation}

\author{
Ali Mobaien \\ Department of Electrical \\ and Computer Engineering \\ Shiraz University \\ Shiraz, Iran \\ Email: a.mobaien@shirazu.ac.ir
}

\author{
Arman Kheirati Roonizi \\ Department of Computer Science \\ Faculty of Science \\ Fasa University \\ Fasa, Iran \\ Email: ebad.kheirati.roonizi@gmail.com
}

\author{
Reza Boostani \\ Department of Electrical \\ and Computer Engineering \\ Shiraz University \\ Shiraz, Iran \\ Email: boostani@shirazu.ac.ir
}

\begin{abstract}
In this work, we present a powerful notch filter for power-line interference (PLI) cancelation from biomedical signals. This filter has a unit gain and a zero-phase response. Moreover, the filter can be implemented adaptively to adjust its bandwidth based on the signal-to-noise ratio. To realize this filter, a dynamic model is defined for PLI based on its sinusoid property. Then, a constrained least square error estimation is used to emerge the PLI based on the observations while the constraint is the PLI dynamic. At last, the estimated PLI is subtracted from recordings. The proposed filter is assessed using synthetic data and real biomedical recordings in different noise levels. The results demonstrate this filter as a very powerful and effective means for canceling the PLI out.
\end{abstract}

Index Terms-Power-line interference, noise cancelation, notch filter, adaptive filter, biomedical recordings.

\section{INTRODUCTION}

Power-line interference (PLI) is one of the major intruder sources while recording biomedical signals such as electroencephalography (EEG) or electrocardiography (ECG). These signals are of great value to determine the health status of individuals and can be used for many clinical or practical purposes (e.g. employing ECG for monitoring heart rate while exercising or recording EEG for brain-computer interfaces [1], [2]). Hence, removing the PLI from biomedical recordings is a very important issue in the field.

The power-line noise is usually attenuated using simple notch filters. These filters are troublous as they do not have unit gain and also the phase response is not constant. Furthermore, these filters are not adapted with the signal-to-noise ratio (SNR) of recordings which may lead to lose information in the situations that the signal power is improved. There are a vast number of studies that have tried to address these problems. In [3], the authors have proposed a feedback scheme to improve the quality factor (Q-factor) of a simple (IIR) second-order notch filter. Although the proposed scheme has improved the Q-factor, the filter has not unit gain and constant phase response. Several adaptive schemes in the literature somehow have tried to modify the Q-factor of the notch filter based on the SNR of recordings [4]. More up-to-date schemes have used the Kalman filter or its extensions to track the PLI and automatically adapt their band-width (i.e. their Q-factor) [5]; however, they require information about the phase and amplitude of the PLI. This is not desirable as these parameters can be easily altered for different recording setups or even within a specific setup (e.g. changing the sensors' impedance due to sweating during recording sessions). In [6], the author has presented a discrete-time linear Kalman filter for PLI cancelation. This filter uses a dynamic model based on trigonometric identities of sinusoid series which doesn't need the PLI amplitude and phase. The methods based on Kalman filtering require an estimation of initial states, they also require the model and observation noises be white. If these conditions are not satisfied, the designed filters might be inaccurate. In these situations, one may use the proposed filtering scheme in [7], which is merely based on minimizing the variance of noise sources.

Contribution: In this work we employ a new approach to design a high Q-factor notch filter for PLI cancelation. This filter has unit gain and zero-phase response to different frequencies. Furthermore, the filter can be implemented adaptively to adjust its bandwidth based on the SNR. The relation of the proposed method with the Wiener filter is also studied, which shows our filter as a generic framework to its Wiener counterpart in the situation of white observation noise. To realize this filter, an additive signal model is defined for observations where the first term is the PLI and the second term is dedicated to desired biomedical signals along with other noise sources. A recursive dynamic model is also defined for the PLI based on the trigonometric properties of sinusoid functions. Owing to this recursive model, there is no need for the amplitude and phase information of the PLI. Now, the observation and dynamic models can be merged to have a good estimation of the PLI. The usual way for this is using a Kalman approach, however, in our model, the observation noise (i.e. the part that is mostly dedicated to the biomedical signals) is highly colored. Hence, here, we utilize a constrained least square error (LSE) estimation [7] to elicit the PLI instead. Finally, the estimated PLI can be subtracted from recordings. The performance of the proposed filter is assessed using synthetic data and real biomedical recordings in different noise levels. The results demonstrate the effectiveness of the proposed filter.

The rest of paper is as follows. In section II, the proposed method is well established. In section III, the Z-domain analysis of the proposed filter, and the relationship of the proposed method with the optimal Winier filter is discussed, also, a scheme is suggested to adapt the filter based on the SNR 
of observations. The performance of the proposed filter for eliminating the PLI from synthetic and real data is assessed in section IV. Finally, the last section is dedicated to a discussion about the proposed method and concludes the paper.

\section{METHOD}

First, we define an additive signal model for observations,

$$
y_{n}=p_{n}+v_{n}, n=1,2, \ldots, N
$$

where $y_{n}$ indicates observations acquired from a recording instrument, $p_{n}$ is the PLI, $v_{n}$ is the noise part assumed to be uncorrelated with $p_{n}, n$ is the time index, and $N$ is the total number of recorded samples. Note that, $v_{n}$ is assumed to be a zero-mean colored noise with variance $\sigma_{v}^{2}$, which contains the desired bio-signals and all other noises (e.g. movement artifacts, etc.).

To define a dynamic model for PLI, this monotonic interference may be considered as,

$$
p_{n}=A \cos \left(\omega_{c} n+\theta\right)
$$

where $A$ and $\theta$ are arbitrary amplitude and phase, respectively. Here, $\omega_{c}=2 \pi f_{c} / f_{s}$ where $f_{c}$ is the frequency of the PLI (usually 50 or $60 \mathrm{~Hz}$ ), and $f_{s}$ is the sampling rate. The model defined in (2) depends on the amplitude and phase of the PLI. This is not desirable since these parameters can be easily changed in different situations. To overcome this problem, one may write (2) as the following recursive equation [6],

$$
p_{n}=\alpha p_{n-1}-p_{n-2}
$$

where $\alpha=2 \cos \left(\omega_{c}\right)$. In the following, a zero-mean white noise $w_{n}$ with variance $\sigma_{w}^{2}$ is added to (3) to address the possible model errors caused by small deviations of the PLI frequency, amplitude or phase.

Putting (1) and (3) together, the following dynamic system is obtained,

$$
\left\{\begin{array}{l}
p_{n}=\alpha p_{n-1}-p_{n-2}+w_{n} \\
y_{n}=p_{n}+v_{n}
\end{array}\right.
$$

To estimate the PLI based on this system, we try to minimize the square error of the dynamic and measurement models simultaneously [7]. This leads to the following constrained LSE optimization problem,

$$
\begin{aligned}
\hat{p}_{n}=\underset{p_{n}}{\arg \min } & \sum_{k=1}^{N}\left(y_{k}-p_{k}\right)^{2} \\
\text { s.t. } & \sum_{k=1}^{N}\left(p_{k}-\alpha p_{k-1}+p_{k-2}\right)^{2} \leq \delta^{2}
\end{aligned}
$$

where $\delta^{2}$ is an upper bound to limit the maximum variation of dynamic model. A matrix form of (5) can be written as,

$$
\begin{array}{cl}
\underline{\hat{p}}=\underset{\underline{p}}{\arg \min } & \|\underline{y}-\underline{p}\|^{2} \\
\text { s.t. } & \left\|H_{c} \underline{p}\right\|^{2} \leq \delta^{2}
\end{array}
$$

where $\|$.$\| indicates the euclidian norm. Here, \underline{p}=$ $\left[p_{1}, p_{2}, \ldots, p_{N}\right]^{T}\left(\{.\}^{T}\right.$ indicates the transpose operator $) ; \underline{y}$ is also defined in the same manner, and it is a column vector of length $N$ consisting the observation samples. $H_{c}$ is a matrix which indicates that the PLI oscillates at $\omega_{c}(\mathrm{rad} / \mathrm{sec})$, and it is defined as,

$$
H_{c}=\left[\begin{array}{cccccc}
1 & -\alpha & 1 & 0 & \cdots & 0 \\
0 & 1 & -\alpha & 1 & \ddots & \vdots \\
\vdots & \ddots & \ddots & \ddots & \ddots & 0 \\
0 & \cdots & 0 & 1 & -\alpha & 1
\end{array}\right]_{(N-2) \times N}
$$

The Lagrangian form of (6) is,

$$
\underline{\hat{p}}=\underset{\underline{p}}{\arg \min }\left\{\|\underline{y}-\underline{p}\|^{2}+\lambda\left\|H_{c} \underline{p}\right\|^{2}\right\} \text {, s.t. } \lambda \geq 0
$$

where $\lambda$ is the Lagrange coefficient. This is a classic LSE problem which is in the form of a convex optimization problem with a quadratic constraint and its solution is obtained by [8], [9],

$$
\underline{\hat{p}}=\left(I+\lambda H_{c}^{T} H_{c}\right)^{-1} \underline{y}
$$

where $I$ is an identity matrix of dimension $N$. In (8), let $\Gamma_{b p f}=\left(I+\lambda H_{c}^{T} H_{c}\right)^{-1}$. One may consider that $\Gamma_{b p f}$ is in fact a bandpass filter which let the PLI to pass. However, we are interested in $\underline{v}$ which indicates the non-PLI part of the observations, including the desired bio-signals. This part is obtained as follows,

$$
\underline{\hat{v}}=\underline{y}-\underline{\hat{p}}=\left(I-\Gamma_{b p f}\right) \underline{y}
$$

where $\Gamma_{n f}=\left(I-\Gamma_{b p f}\right)$ is the final form of the proposed notch filter. There is only one parameter to be set in this filter, and it is $\lambda$. Increasing $\lambda$ from zero force the $\underline{\hat{p}}$ to be more like a sinusoid series, and in the sense of a notch filter, it means a narrower stop-band. In the next section we discuss how changing the $\lambda$ value changes the $\mathrm{Q}$-factor of the filter.

\section{Properties of the Proposed Filter}

\section{A. Z-domain Analysis}

The sample-wise form of (8) can be written as,

$$
\hat{p}_{n}=\left(\gamma_{n}^{b p f}\right)^{-1} * y_{n}
$$

In (10), $\gamma_{n}^{b p f}=\left(\delta_{n}+\lambda h_{-n} * h_{n}\right)$ where $\delta_{n}$ is the Kronecker delta, $h_{n}=[1,-\alpha, 1]$, and $*$ represents the convolution operator. This equation means that $\hat{p}_{n}$ is obtained by the deconvolution of $\gamma_{n}^{b p f}$ and $y_{n}$ (or equivalently $\gamma_{n}^{b p f} * \hat{p}_{n}=y_{n}$ ). $\gamma_{n}^{b p f}$ represents a linear time-invariant (LTI) and non-causal bandpass filter with the following transfer function,

$$
\Gamma^{b p f}(z)=\frac{1}{1+\lambda \gamma^{b p f}\left(z^{-1}\right) \gamma^{b p f}(z)}
$$

where $\gamma^{b p f}(z)=1-\alpha z^{-1}+z^{-2}$. By the same logic and employing (9), the Z-transform of the notch filter is obtained as,

$$
\Gamma^{n f}(z)=1-\frac{1}{1+\lambda \gamma^{b p f}\left(z^{-1}\right) \gamma^{b p f}(z)}
$$



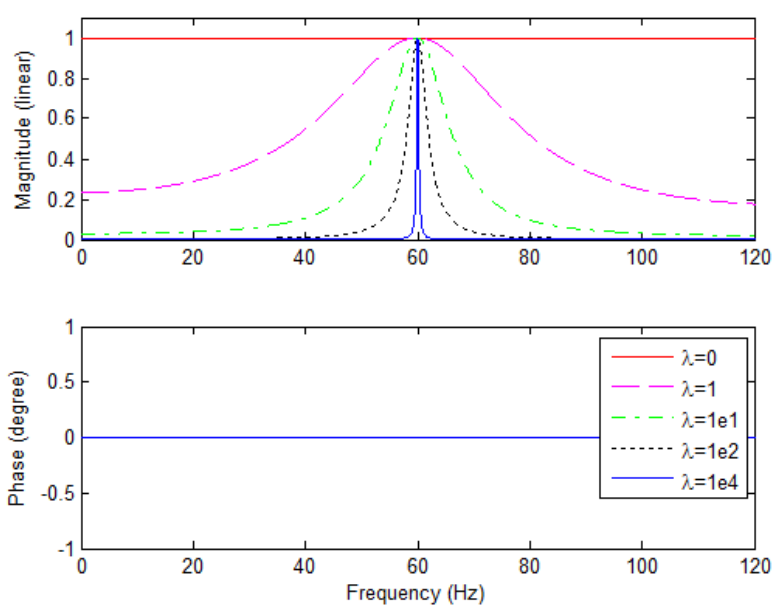

Fig. 1. The amplitude and phase response of the proposed bandpass filter in different values of $\lambda . \lambda=0,1,1 \mathrm{e} 1,1 \mathrm{e} 2$, and $1 \mathrm{e} 4$ from top to bottom. The phase response for all $\lambda$ values is zero, hence, the plots are overlapped.
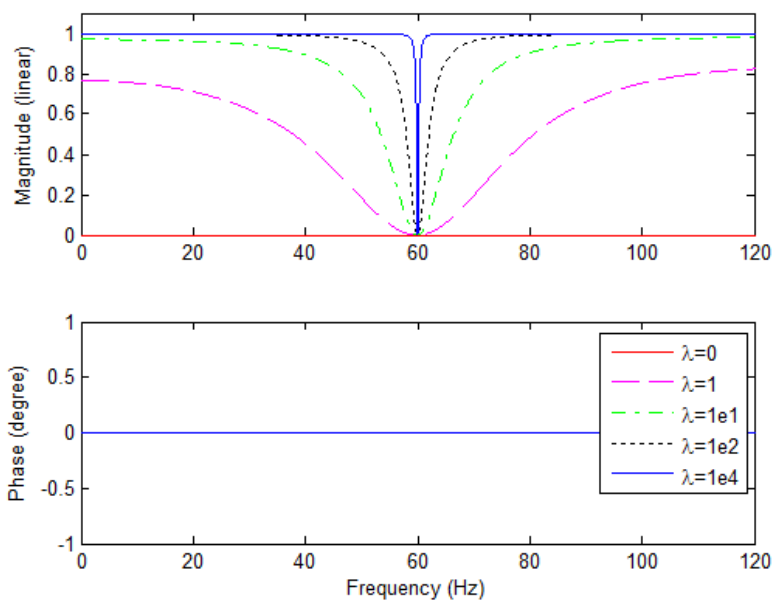

Fig. 2. The amplitude and phase response of the proposed notch filter in different values of $\lambda . \lambda=0,1,1 \mathrm{e} 1,1 \mathrm{e} 2$, and $1 \mathrm{e} 4$ from top to bottom. The phase response for all $\lambda$ values is zero, hence, the plots are overlapped.

Figs. 1 and 2 show the frequency response of the proposed bandpass and notch filters for $f_{c}=60 \mathrm{~Hz}$ and $f_{s}=256 \mathrm{~Hz}$. As it can be seen, they both have a unit gain and zero phase response. By increasing the value of $\lambda$ from zero, the Q-factor of the filters is increased.

\section{B. Relation with the Winier Filter}

Winier filter is the optimal solution for estimating a desired process by LTI filtering of noisy observations. It is assumed that signal and noise come from stationary processes with known spectra, and the noise is additive [10]. Considering (1), the transfer function of the Winier filter minimizing the mean square error between the estimated process and the desired process is obtained by,

$$
\Gamma^{W i n i e r}(z)=\frac{S_{p p}(z)}{S_{p p}(z)+S_{v v}(z)}
$$

where $S_{p p}(z)$ and $S_{v v}(z)$ are the spectra of $p_{n}$ and $v_{n}$, respectively. By considering the defined dynamic system in (4) and assuming that $v_{n}$ comes from a white random process, we have,

$$
\begin{gathered}
\Gamma^{\text {Winier }}(z)=\left(\frac{\frac{\sigma_{w}^{2}}{\gamma^{b p f}\left(z^{-1}\right) \gamma^{b p f}(z)}}{\frac{\sigma_{w}^{2}}{\gamma^{b p f}\left(z^{-1}\right) \gamma^{b p f}(z)}+\sigma_{v}^{2}}\right) \\
=\frac{1}{1+\frac{\sigma_{v}^{2}}{\sigma_{w}^{2}} \gamma^{b p f}\left(z^{-1}\right) \gamma^{b p f}(z)}
\end{gathered}
$$

which is equal to (11) if $\lambda=\sigma_{v}^{2} / \sigma_{w}^{2}$. Hence, we can consider the proposed filter as a generic form of the Winier filter when the observation noise comes from a white process.

\section{Adaptive Form of the Proposed Filter}

The only tunable parameter of the proposed notch filter is $\lambda$. If $\lambda$ is set to a specific value, the filter will have a fixed frequency response and a fixed stop-band. However, it is desired to have a narrower stop-band for high SNR situations to let more of the desired signal passes through the filter, and, it is the other way for low SNR situations. Hence, it is insightful to consider $\lambda$ proportional to the SNR. To have an estimation of the SNR, one may pass the observation vector from a coarse notch filer and calculate the power (variance) of the filtered signal. Let $\hat{\sigma}_{v}^{2}$ be an estimation of the desired signal power, and let $\hat{\sigma}_{P}^{2}$ be an estimation of the PLI power $\left(\hat{\sigma}_{P}^{2}\right.$ can be obtained by subtracting the filtered signal from observations and calculating the variance of the residue). Now, the adaptive $\lambda$ value is calculated by,

$$
\lambda_{\text {adaptive }}=C \frac{\hat{\sigma}_{v}^{2}}{\hat{\sigma}_{P}^{2}}
$$

where $C$ is a constant scale parameter.

Another approach to find an adaptive value for $\lambda$ is plotting an L-curve [11] and finding the best value (which is corresponding to the corner point of the L-curve). This gives the best compromise between following the sinusoid dynamic and following the observations. It can be seen that for higher SNRs, the corner point corresponds to a larger $\lambda$, and it is vice versa for lower SNRs.

\section{Method Evaluation}

In this section, the proposed notch filter is evaluated for PLI cancelation from synthetic and real data. For real data, ECG and EEG signals are employed. The results are presented in the three following forms. First, the performance of the proposed filter in the time domain, second, its performance in the frequency domain by employing power spectral density (PSD) plots, and at last, the mean square error (MSE) between the desired signal and filtered signal in different SNRs are presented. For all experiments, $\lambda$ is set to $1 \mathrm{e} 4$. 
(a)

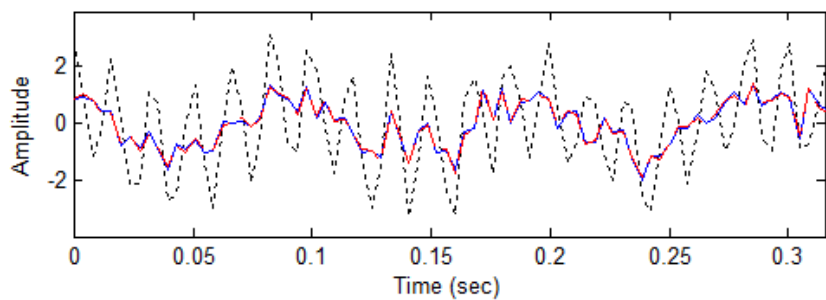

(b)

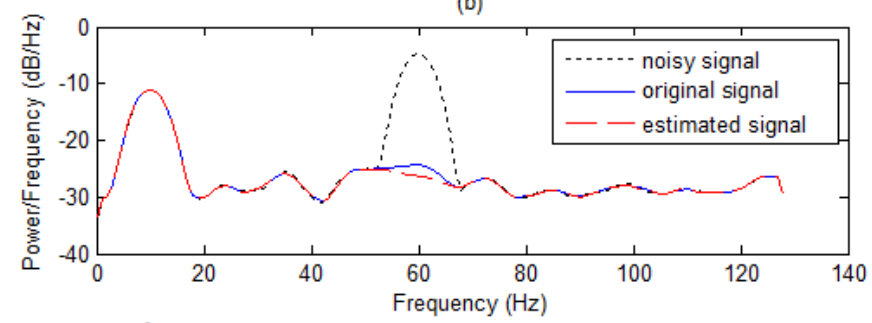

(c)

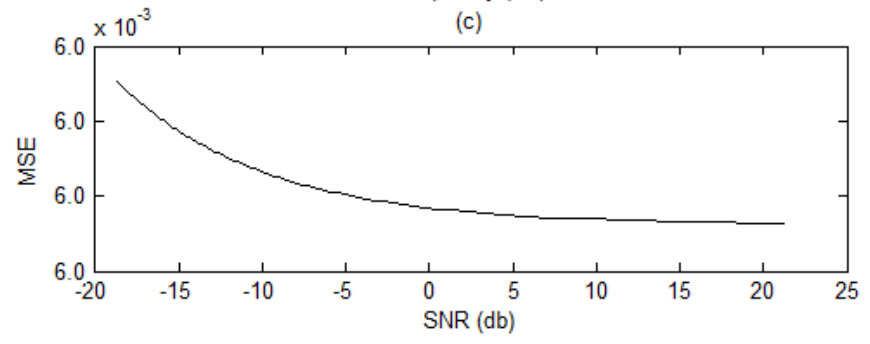

Fig. 3. The performance of the proposed notch filter for removing the PLI from synthetic data. (a) The temporal templates of noisy, original, and estimated signals. (b) The PSD of noisy, original, and estimated signals. (c) The MSE between the original and estimated signals for different SNRs.

\section{A. Synthetic Data}

For synthetic data, we generate a random sequence contaminated by the PLI $(60 \mathrm{~Hz})$. Consider $y_{n}=2 \cos \left(\omega_{c} n\right)+$ $\cos \left(\omega_{0} n+\pi / 6\right)+r_{n}$ as noisy observations. Here, $\omega_{0}$ corresponds to $10 \mathrm{~Hz}, r_{n}$ is a white zero-mean Gaussian sequence with variance 0.25 , and $f_{s}=256 \mathrm{~Hz}$. Fig. 3 shows the performance of the proposed notch filter for removing PLI from synthetic data. In Fig.3a the signals in time domain are shown. As it can be seen, the PLI is effectively canceled and there is no phase difference between the noiseless (original) and filtered (estimated) signals, and the estimated signal is very close to its original version. The PSD of the signals can be found in Fig.3b. There is a pick at $60 \mathrm{~Hz}$ in the PSD of the noisy observations, however, this pick is significantly mitigated for filtered data. One may consider that the PSD of the original signal is slightly higher than the filtered signal at the PLI frequency. This is due to the existence of some $60 \mathrm{~Hz}$ components in $r_{n}$ which are removed from the estimated sequence. Fig. $3 c$ represents the MSE between the desired signal and filtered signal in different SNRs. It can be seen that even in very low SNR situations, the MSE is very small. However, by increasing the SNR the MSE is decreased, as expected. (a)

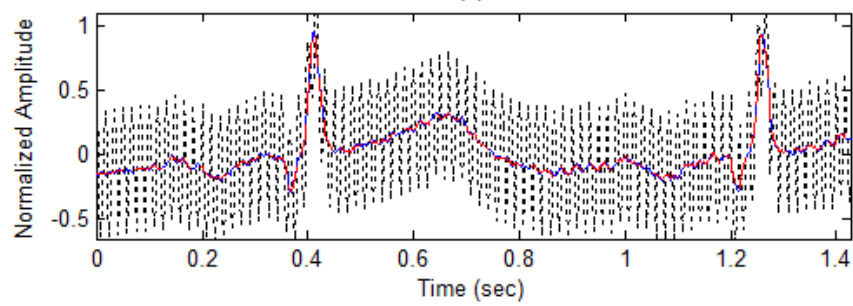

(b)

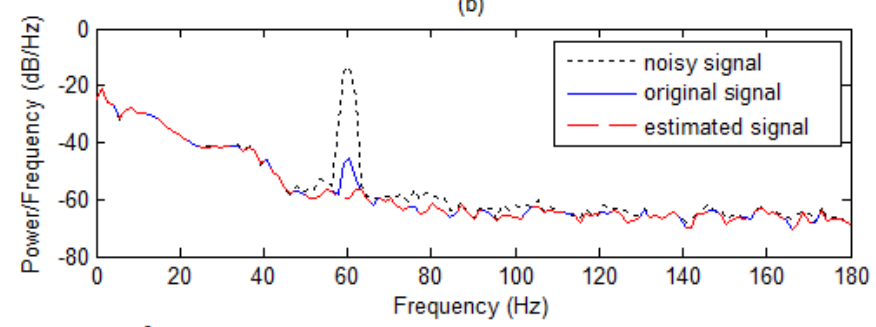

(c)

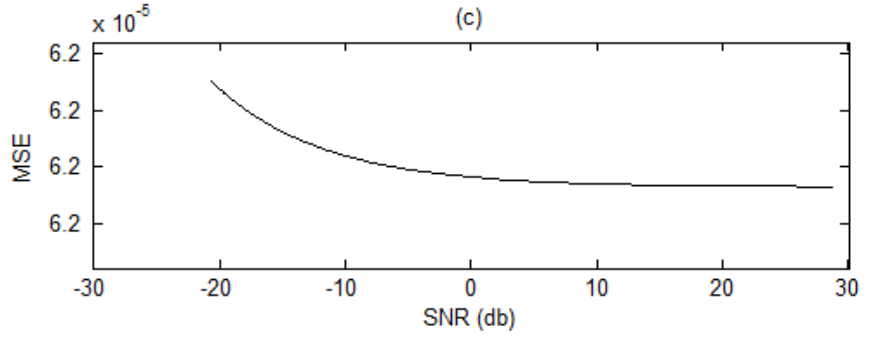

Fig. 4. The performance of the proposed notch filter for removing the PLI from a real ECG sequence (sampled at $360 \mathrm{~Hz}$ ). (a) The temporal templates of noisy, original, and estimated signals. (b) The PSD of noisy, original, and estimated signals. (c) The MSE between the original and estimated signals for different SNRs.

\section{B. ECG Data}

Here, a sequence of normal ECG is employed to assess the performance of the proposed notch filter. The original ECG signal is obtained from a publicly available dataset [12], and it is demeaned and normalized. Then, this template is contaminated by a synthetic PLI to generate noisy observations. Fig.4 represents the results for ECG data. Same as the former case, the PLI is effectively removed from the ECG sequence without causing any delay (Fig.4a). In the PSD plots (Fig.4b), it can be seen that the noise pick at $60 \mathrm{~Hz}$ is effectively suppressed for the filtered signal. Note that, in the PSD of the original signal, there is a small pick at $60 \mathrm{~Hz}$ which can be related to an unsuppressed PLI. However, in the PSD of the estimated signal, this pick is significantly mitigated. Fig.4c shows the MSE between the desired signal and filtered signal in different SNRs. Here again, it can be seen that the MSE is very small, and by increasing the SNR the MSE is decreased.

\section{EEG Data}

To investigate the performance of our proposed method for PLI cancelation from EEG data, here, an EEG signal from a publicly available dataset [13] is used. This signal is demeaned and normalized to obtain the original signal, then, it is contaminated by a synthetic PLI to generate noisy observations. Fig.5 shows the results for EEG data filtering. 
(a)

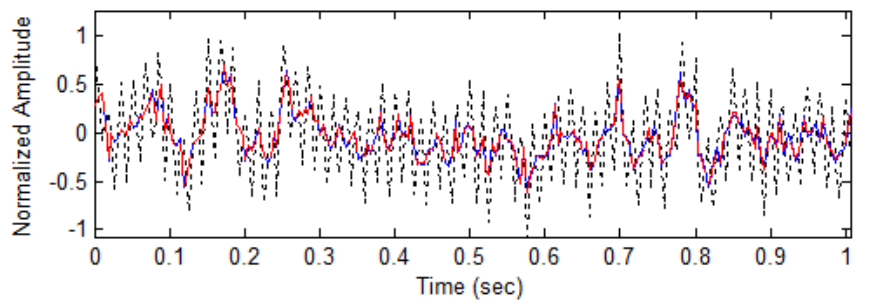

(b)
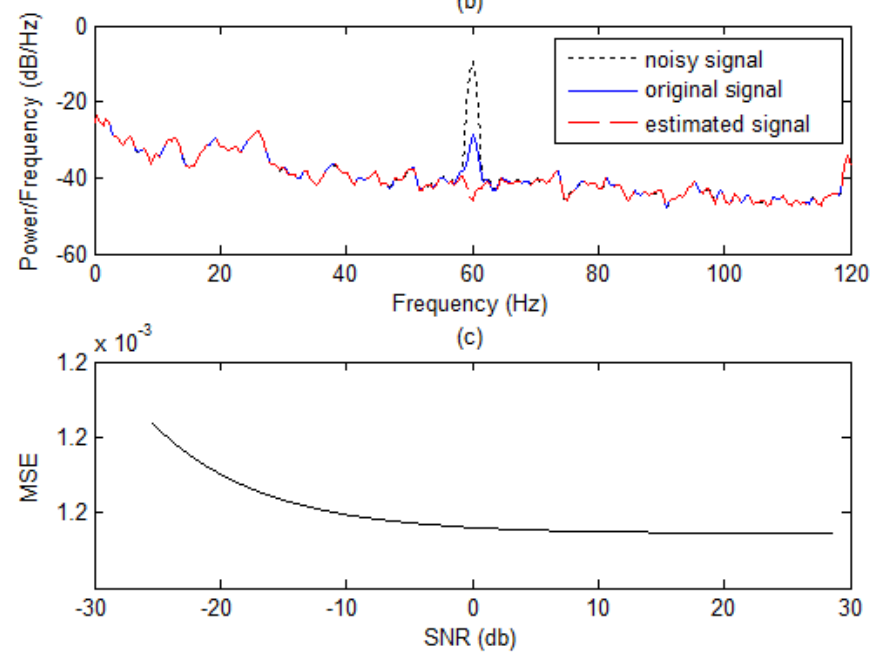

Fig. 5. The performance of the proposed notch filter for removing the PLI from a real EEG sequence (sampled at $240 \mathrm{~Hz}$ ). (a) The temporal templates of noisy, original, and estimated signals. (b) The PSD of noisy, original, and estimated signals. (c) The MSE between the original and estimated signals for different SNRs.

Same as the two former cases, the PLI is effectively suppressed and there is no time delay between the original and estimated signals (Fig.5a). Fig.5b represents the PSD plots. It can be seen that the noise pick at $60 \mathrm{~Hz}$ is effectively attenuated for the filtered signal. Same as the ECG data, in the PSD of the original EEG signal, there is a small pick at $60 \mathrm{~Hz}$ which can be related to an unsuppressed PLI. However, in the PSD of the estimated signal, this pick is mitigated. The MSE between the desired signal and filtered signal in different SNRs is presented in Fig.5c. Same as before, it can be seen that the MSE is very small, and by increasing the SNR it is decreased even more.

\section{Discussion AND CONCLUSION}

In this study, a powerful notch filter is presented which can effectively remove the power-line interference from different biomedical recordings. This filter has unit gain and zero phase in response to different frequencies. The Q-factor of the filter can be simply altered (by modifying the $\lambda$ value) while its amplitude and phase response properties are preserved. This is important since designing high Q-factor notch filters is difficult, and their frequency response may be unstable. For example, in [3], a feedback scheme has been used to implement a high-quality filter, however, the filter does not have unit gain and constant phase response.
The proposed filter can be implemented adaptively to accord with the SNR. A popular method for achieving adaptive notch filters is through Kalman filtering. Many studies have used the Kalman filter or its extensions to track the PLI [5], however, they usually require the phase and amplitude information. This is not desirable as these parameters are not fixed in different situations. In comparison, our method doesn't need the amplitude or phase of the PLI, thanks to a recursive dynamic model based on the intrinsic property of the sinusoid series. Another point that should be noted is that the Kalman filter requires a good estimation of initial states and also white noises for observation and dynamic models to perform accurately. This may be limiting even using a Kalman notch filter which doesn't need the amplitude and phase information [6]. In our study, this is solved by employing a constrained LSE estimation, instead. This method tries to minimize the square error of the dynamic and measurement models simultaneously. The performance of the proposed filter is assessed using synthetic data and real biomedical signals in different noise levels. It is demonstrated that the PLI is effectively suppressed in all cases.

In this study, no dynamic model is assumed for biomedical signals. Assuming a dynamic model for the non-PLI part of the observations may improve the purposed method and should be studied in future work.

\section{REFERENCES}

[1] M. Dehghani, A. Mobaien, and R. Boostani, "A deep neural networkbased transfer learning to enhance the performance and learning speed of BCI systems," Brain-Computer Interfaces, vol. 8, no. 1-2, pp. 14-25, 2021.

[2] A. Mobaien and R. Boostani, "ACSP: Adaptive CSP filter for BCI applications," in 2016 24th Iranian Conference on Electrical Engineering (ICEE). IEEE, 2016, pp. 466-471.

[3] C.-C. Tseng and S.-C. Pei, "Stable IIR notch filter design with optimal pole placement," IEEE Trans. Signal Process., vol. 49, no. 11, pp. 2673 2681, 2001.

[4] J. Glover, "Adaptive noise canceling applied to sinusoidal interferences," IEEE Trans. Acoust., Speech, Signal Process., vol. 25, no. 6, pp. 484 491, 1977.

[5] L. Avendano-Valencia, L. Avendano, J. Ferrero, and G. CastellanosDominguez, "Improvement of an extended Kalman filter power line interference suppressor for ECG signals," in 2007 Computers in Cardiology. IEEE, 2007, pp. 553-556.

[6] R. Sameni, "A linear Kalman notch filter for power-line interference cancellation," in The 16th CSI International Symposium on Artificial Intelligence and Signal Processing (AISP 2012). IEEE, 2012, pp. 604610.

[7] A. K. Roonizi, "A new approach to ARMAX signals smoothing: Application to variable-Q ARMA filter design," IEEE Trans. Acoust., Speech, Signal Process., vol. 67, no. 17, pp. 4535-4544, 2019.

[8] S. P. Boyd and L. Vandenberghe, Convex Optimization. Cambridge University Press, 2004.

[9] W. Gander, "Least squares with a quadratic constraint," Numer. Math. (Heidelb), vol. 36, no. 3, pp. 291-307, 1980

[10] N. Wiener et al., Extrapolation, Interpolation, and Smoothing of Stationary Time Series: With Engineering Applications. MIT press Cambridge, MA, 1964, vol. 8 .

[11] P. C. Hansen, "The L-curve and its use in the numerical treatment of inverse problems," IMM, Department of Mathematical Modelling, Technical University of Denmark, 1999.

[12] P. Plawiak, "ECG signals (1000 fragments)," Mendeley Data, v3, 2017.

[13] M. Kaper, P. Meinicke, U. Grossekathoefer, T. Lingner, and H. Ritter, "BCI competition 2003-data set IIb: Support vector machines for the P300 speller paradigm," IEEE Trans. Biomed. Eng., vol. 51, no. 6, pp. 1073-1076, 2004. 